\title{
Feasibility Assessment of Support Vector Regression Models with Immune Algorithms in Predicting Fatigue Life of Composites
}

\author{
Wei-Chiang Hong ${ }^{1}$ Feng-Min Lai ${ }^{2}$ Jia-Hroung Wu ${ }^{3}$ Ping-Feng Pai $^{4^{*}}$ Shun-Lin Yang ${ }^{2}$ \\ Department of Information Management, Oriental Institute of Technology \\ No. 58, Sec. 2, Si-Chuan Rd., Pan-Chiao, Taipei, 220, Taiwan \\ 2 Department of Industrial Engineering and Technology Management, Da-Yeh University \\ 112 Shan-Jiau Rd., Da-Tusen, Changhua, 51505, Taiwan \\ ${ }^{3}$ Department of Industrial Management, Hsiuping Institute of Technology \\ 11 Industrial Rd., Dali City, Taichung County 41180, Taiwan \\ ${ }^{4}$ Department of Information Management, National Chi Nan University \\ 1 University Rd., Puli, Nantou, 545, Taiwan \\ paipf@ncnu.edu.tw
}

\begin{abstract}
Predicting fatigue life of composite materials is essential to increase reliability of manufacturing systems. The predicting techniques for fatigue life of composite materials are not widely investigated. The support vector regression (SVR) is an emerging forecasting technique and has been applied in many areas successfully. Therefore, this study attempts to examine the feasibility of SVR in predicting the fatigue life of composite materials. Additionally, immune algorithms (IA) are used to select three parameters of SVR models. An experimental data set from a laboratory was employed to depict the feasibility of develpoed SVRIA (support vector regression with immune algorithms) approach in predicting fatigue life of composite materials. Empirical results indicate that the SVRIA is a valid way in predicting fatigue life of composite materials.
\end{abstract}

Keywords: Support vector regression; immune algorithms; fatigue life prediction; composite materials

\section{Introduction}

Composite materials have been widely used in automotive and aerospace applications due to the high stiffness and strength [1]. Using composite materials in industry can reduce life-cycle costs and save potential energy [2]. However, most of these applications are subjected to cyclic fatigue loading, which can easily cause material damage, and finally structural breakage. Therefore, predicting fatigue life of composite materials is crucial in fatigue-resistant design. The analysis is to predict the remaining life of composite materials based on previous loading historical data. The fatigue damage of composite materials involves many mechanisms. Therefore, the fatigue life prediction is a complex process with nonlinear characteristics [3][4].

Artificial neural networks (ANN) is an effective method to predict the remaining life of composite materials. Artymiak et al. [5] applied ANN to predict finite-life fatigue strength and fatigue limit of steel. They reported that the ANN model is superior to the other approaches in terms of prediction performance. Venkatesh and Rack [6] presented a back-propagation neural network to predict the fatigue life. The authors found that the proposed neural network can estimate elevated temperature creep-fatigue life of the Ni-based alloy INCONEL 690. Pleune and Chopra [7] used back-propagation neural networks to predict the fatigue life of carbon and low-alloy steels for specified sets of loading and environmental conditions. It was reported that proposed ANN model predicts the fatigue life accurately with incomplete data sets. AlAssaf and El Kadi [8] proposed a feedforward neural network to determine the relationship between the input parameters and the fatigue life of a unidirectional glass fiber/epoxy composite laminate. The simulation results obtained by the proposed ANN model are comparable to existing fatigue life prediction models. El Kadi and Al-Assaf [9] compared the performance of different neural networks in predicting the fatigue life of unidirectional glass fiber/epoxy composite laminate. They claimed that the modular neural networks outperformed the other models in terms of prediction accuracy. El Kadi and Al-Assaf [10] used a modular neural network to 
predict fatigue life of fiberglass/epoxy composites. The experimental results showed that the modular neural network with the strain energy resulted in the best prediction of fatigue life. Choi et al. [11] predicted the fatigue damage growth in composite laminates by ANN. They pointed out that the presented neural network has more accurate prediction results than the power law model.

Developed by Vapnik [12], the support vector machine is a novel neural network algorithm and originally applied to pattern recognition problems. The SVR [13] technique has been investigated to deal with non-linear regression problems and has provided many exciting prediction results in the field of finance[14], air quality[15], wind speed[16], reliability[17], electricity load [18], and tourist arrivals. Thus, the aim of this work is to study the feasibility of SVRIA in predicting the fatigue life of composites.

\section{Support Vector Regression with Immune Algorithms}

Instead of searching empirical errors, SVR technique minimizes an upper bound of the generalization error [12]. SVR map nonlinearly the original data $x$ into a higher dimensional feature space. In such a way, the data not being divided by a linear function in the input space can be partitioned in the feature space. The linear function $g(x)=w x+u$ is always used to conduct the regression in the feature space. The coefficients, $w$ and $u$, are determined by the following equation:

M in : $R(f)=C \sum_{i=1}^{N} L_{\varepsilon}\left(v_{i}, g_{i}\right)+\frac{1}{2}\|w\|^{2}$

with the following constraints:

$$
\begin{aligned}
& w x_{i}+u-v_{i} \leq \varepsilon+\xi_{i}^{*}, \\
& v_{i}-w x_{i}-u \leq \varepsilon+\xi_{i}, \\
& \xi_{i}, \xi_{i}^{*} \geq 0, i=1,2, \ldots, \mathrm{N}
\end{aligned}
$$

The first part Eq(1) indicates forecasting errors larger than $\pm \varepsilon$ are denoted with two slack variables, $\xi$ (above $\varepsilon$ ) and $\xi^{*}$ (below $\varepsilon$ ) correspondingly. The second part measures the flatness of the function, which is as small as possible. In addition, both $\mathrm{C}$ and $\varepsilon$ are parameters determined by users.

Eq.(1) can be solved by performing Lagrangian theory and derived the weight vector, $w$, expressed as Eq.(5):

$w=\sum_{i=1}^{N}\left(\beta_{i}-\beta_{i}^{*}\right) x_{i}$

where $\beta_{i}$ and $\beta_{i}^{*}$ are Lagrange multipliers and the asterisks denote the statuses above or below the regression line. Then, Eq.(5) is reformulated as Eq.(6).

$g(x)=\sum_{i=1}^{N}\left(\beta_{i}-\beta_{i}^{*}\right)\left\langle x_{i}, x\right\rangle+u$ where $\left\langle x_{i}, x\right\rangle$ represents the inner product.

In Eq.(6), $x_{i}$ could be replaced by a mapping to feature space, $\phi\left(x_{i}\right)$. Therefore, Eq.(6) can be expressed as following equation:

$$
g(x)=\sum_{i=1}^{N}\left(\beta_{i}-\beta_{i}^{*}\right) K\left(x_{i}, x\right)+u
$$

where $K\left(x_{i}, x\right)=\left\langle\phi\left(x_{i}\right), \phi(x)\right\rangle \cdot K\left(x_{i}, x\right)$ is a Kernel function and the value of a Kernel equals to inner product of two vectors, $\phi\left(x_{i}\right)$ and $\phi(x)$. Gaussian kernel function, represented as Eq.(8), is used in this study.

$$
K\left(x_{i}, x\right)=\exp \left(-\left\|x_{i}-x\right\|^{2} / 2 \sigma^{2}\right)
$$

Thus, a SVR model contains three parameters: $\sigma, \varepsilon$ and $C$. The selection of three parameters influences the forecasting accuracy of a SVR model a lot. In this study, the IA is used to seek a better combination of the three parameters of SVR so that a smaller prediction error can be obtained.

IA [20] was developed on the basis of natural immune systems that efficiently recognize all cells within the body and classify those cells as self or nonself cells. The non-self cells stimulate a defensive mechanism to defense foreign invaders, such as bacteria and viruses. In this study, antibodies are represented by three SVR parameters. The prediction error of SVR is contained in the denominator part of the affinity formula. Therefore, maximizing the affinity of IA implies the minimization of prediction errors of SVR model. The mean absolute percentage error (MAPE), given by (9), is used to calculate predicting errors.

$M A P E=\frac{1}{N} \sum_{i=1}^{N}\left|\frac{Y_{i}-y_{i}}{Y_{i}}\right| \times 100 \%$

where $N$ represents the number of experiments ; $Y_{i}$ is the actual value of experiment $i$; and $y_{i}$ denotes the predicting value of experiment $i$. The framework of SVRIA model is depicted in Fig. 1. More detail of applying IA in selecting SVR parameters is depicted as follows.

Step 1: Initializing the antibody population randomly Binary-code strings containing three parameters ( $\sigma$, $C$, and $\varepsilon$ ) of a SVR model is used to represented the antibody population. The initial antibody population is created randomly. For instance, suppose that an antibody contains nine binary codes representing three SVR parameters. Each parameter is thus expressed by three binary codes. Additionally, assume that the limits of $\sigma, C$, and $\varepsilon$ are 1,100 , and 0.8 correspondingly. The antibody with binary-code "101001110" implies that the values of the three parameters $\sigma, C$, and $\varepsilon$ are $0.714,57.14$, and 0.343 respectively. The number of initial antibodies is the same as the size of the memory cell set to 20 in this study.

Step 2: Identifying the affinity and the similarity 
Antibodies have higher activation levels of antigens when the affinity values are high. To maintain the diversity of the antibodies stored in the memory cells, an antibody with a higher affinity value and a lower similarity value has a good likelihood to enter the memory cells. The affinity between the antibody and antigen is defined as follows:

$A g_{k}=1 /\left(1+M A P E_{k}\right)$

A smaller prediction error (MAPE) indicates a larger value of affinity. The similarity between antibodies is expressed as Eq. (11).

$A b_{i j}=1 /\left(1+T_{i j}\right)$

where $T_{i j}$ represents the difference between the two SVR forecasting errors calculated by the antibodies inside and outside memory cells.

Step 3: Selecting antibodies in memory cells

Antibodies with higher values of $A g_{k}$ are viewed as candidates to enter the memory cell. However, the antibody candidates with $A b_{i j}$ values exceeding a threshold are not qualified to enter the memory cell. In this study, the threshold value is set to 0.85 .

Step 4: Performing crossover and mutation of antibody population.

Crossover and mutation are used to generate new antibodies. When conducting crossover operation, strings representing antibodies are paired randomly. Segments of paired strings between two predetermined break-points are swapped. The mutation operation randomly converts a code " 0 " into " 1 ", or a code " 1 " into a " 0 ". The crossover and mutation rates are determined using probabilities.

Step 5: Stopping criteria

When the number of iterations reaches a given scale, the best antibody is a solution. Otherwise, return to Step 2.

\section{A Numerical Example}

Experimental data of fatigue life provided by the Composite Materials \& Structure Laboratory at National Chiao Tung University (Taiwan) waere used to examine the feasibility of the proposed SVRIA model in fatigue life prediction. To obtain the properties and strengths of the laminated composite specimens, six types specimens were employed. The specimens were mounted and tested in a properly aligned and calibrated INSTRON 8801 test machine. Wedge action friction grips of hydraulic grips were used with the crosshead rate set at $1 \mathrm{~mm} / \mathrm{min}$.

After each specimen was finished fatigue testing, the tensile experiment was conducted. From fatigue testing, parameters of the different fiber orientation and width of specimens provide the residual strength and residual Young's modulus decay. The experiment with more than 100,000 cyclic loading periods was conducted. Twenty residual stiffness degradation ratios for each specimen were obtained. The data set was divided into three parts, the training data, the validation data, and the testing data. The numbers of training set, validation data set, and testing data set are ten, five, and five respectively. In this investigation, the rolling-based prediction procedure and a one-stepahead prediction policy are employed in this experiment.

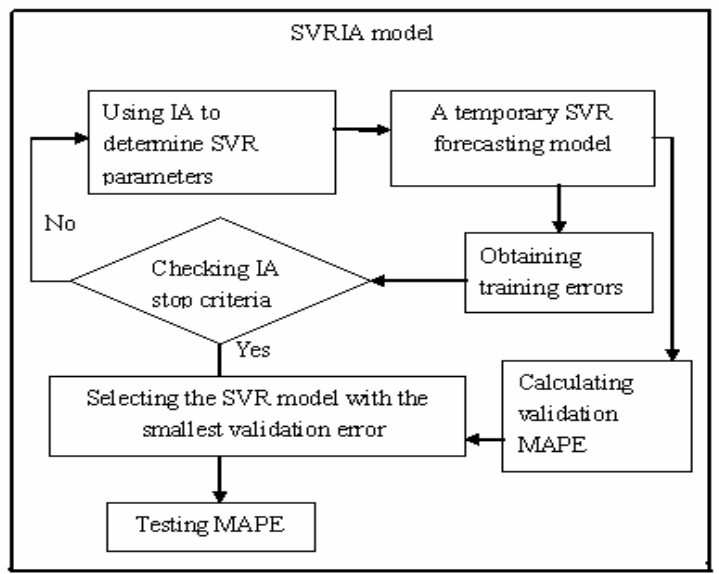

Fig. 1 The SVRIA framework

Table 1. Testing MAPE values of six specimen and parameters of three prediction models

\begin{tabular}{|c|c|c|c|}
\hline $\begin{array}{l}\text { Types of } \\
\text { specimens }\end{array}$ & SVRIA & ARIMA & GRNN \\
\hline$\left[0_{4}\right]_{S}$ & $\begin{array}{l}(\sigma, \mathrm{C}, \varepsilon)= \\
(0.82,74.62 \\
0.09) ; \\
\text { MAPE }=1.39\end{array}$ & $\begin{array}{l}(p, d, q)= \\
(1,1,1) ; \\
\text { MAPE= } \\
6.72\end{array}$ & $\begin{array}{c}\sigma=0.04 ; \\
\text { MAPE }=6.42\end{array}$ \\
\hline$\left[0_{2} / 90_{2}\right]_{S}$ & $\begin{array}{l}(\sigma, \mathrm{C}, \varepsilon)= \\
(0.88, \quad 47.66, \\
0.11) ; \\
\text { MAPE }=1.69\end{array}$ & $\begin{array}{l}(p, d, q)= \\
(1,2,1) ; \\
\text { MAPE= } \\
3.23\end{array}$ & $\begin{array}{l}\sigma=0.03 ; \\
\text { MAPE=5.72 }\end{array}$ \\
\hline $\begin{array}{l}{\left[0_{2} / 45 /-\right.} \\
45]_{S}\end{array}$ & $\begin{array}{l}(\sigma, \mathrm{C}, \varepsilon)= \\
(0.98, \quad 5.16, \\
0.02) ; \\
\text { MAPE }=2.40\end{array}$ & $\begin{array}{l}(p, d, q)= \\
(1,2,1) ; \\
\text { MAPE= } \\
6.21\end{array}$ & $\begin{array}{l}\sigma=0.05 ; \\
\text { MAPE }=4.57\end{array}$ \\
\hline $\begin{array}{l}{\left[0{ }_{2} / 45 /-\right.} \\
45]_{S}\end{array}$ & $\begin{array}{l}(\sigma, \mathrm{C}, \varepsilon)= \\
(0.35,50.78, \\
0.67) ; \\
\text { MAPE }=3.34\end{array}$ & $\begin{array}{l}(p, d, q)= \\
(1,0,1) ; \\
\text { MAPE= } \\
8.57\end{array}$ & $\begin{array}{c}\sigma=0.02 ; \\
\text { MAPE }=5.79\end{array}$ \\
\hline $\begin{array}{l}90 / 45 /- \\
45 / 0]_{\mathrm{S}}\end{array}$ & $\begin{array}{l}(\sigma, \mathrm{C}, \varepsilon)= \\
(0.74, \quad 64.84, \\
0.13) ; \\
\text { MAPE }=1.73\end{array}$ & $\begin{array}{l}(p, d, q)= \\
(1,2,1) ; \\
\text { MAPE= } \\
5.29\end{array}$ & $\begin{array}{l}\sigma=0.06 ; \\
\text { MAPE=5.68 }\end{array}$ \\
\hline $\begin{array}{l}{[45 /-} \\
45 / 45 /- \\
45]_{\mathrm{S}}\end{array}$ & $\begin{array}{l}(\sigma, \mathrm{C}, \varepsilon)= \\
(0.88, \quad 30.86, \\
0.09) ; \\
\text { MAPE }=2.65\end{array}$ & $\begin{array}{l}(p, d, q)= \\
(1,2,1) ; \\
\text { MAPE= } \\
6.40\end{array}$ & $\begin{array}{c}\sigma=0.03 \\
\text { MAPE }=5.56\end{array}$ \\
\hline
\end{tabular}

The model with the minimum validation MAPE value for each experiment is selected as the most appropriate model in this study. Two other prediction models, namely autoregressive integrated moving average (ARIMA) [21] and general regression neural networks (GRNN) [22], are used to predict the same experimental data of fatigue life. The MAPE values 
for six specimens of three models and corresponding parameters are shown in Table 1.

\section{Conclusions}

The fatigue life of composite materials is the remaining life of a structure expressed as a time-series loading data. To prevent the break of materials in advance, accurate estimation of fatigue lie is essential. This study develops a SVRIA model and verifies its feasibility in predicting the fatigue life of composites. Besides, the SVRIS model outperforms the ARIMA model and the GRNN model in terms of prediction accuracy. For the future research, some other factors causing the fatigue of composite materials such as material type can be considered in the prediction model.

\section{Acknowledgements}

This research was conducted with the support of National Science Council (94-2213-E-260-023). The experiment data were provided by Professor JiaHroung Wu from the Composite Materials \& Structure Laboratory at National Chiao Tung University, Taiwan.

\section{References}

[1] D.H. Middleton, "Composite Materials in Aircraft Structures”, John Wiley, New York, 1990.

[2] R.M. Jones, "Mechanics of Composite Materials", Edwards Brothers, Ann Arbor, MI ,1999.

[3] L. J. Lee, K.E. Fu, and J.N. Yang, "Prediction of Fatigue Damage and Life for Composite Laminates under Service Loading Spectra”, Composite Science and Technology, Vol. 56, pp. 635-648, 1996.

[4] M.A. Miner, "Cumulative Damage in Fatigue", Journal of Applied Mechanics, Vol. 12. pp. A159A164, 1945.

[5] P. Artymiak, L. Bukowski, J. Feliks, S. Narberhaus, and H. Zenner, H., "Determination of S-N Curves with the Application of Artificial Neural Networks", Fatigue and Fracture of Engineering Materials and Structures, Vol. 22 , pp. 723-728, 1999.

[6] V. Venkatesh, H.J. Rack, "A Neural Network Approach to Elevated Temperature Creep-Fatigue Life Prediction", International Journal of Fatigue, Vol. 21, pp. 225-234, 1999.

[7] T.T. Pleune, O.K. Chopra, "Using Artificial Neural Networks to Predict the Fatigue Life of Carbon and Low-Alloy Steels”, Nuclear Engineering And Design, Vol. 197, pp. 1-12, 2000.

[8] Y. Al-Assaf, H. El Kadi,. "Fatigue Life Prediction of Unidirectional Glass Fiber/Epoxy Composite
Laminate Using Neural Networks”, Composite Structures, Vol. 53, pp. 65-71, 2001.

[9] H. El Kadi, Y. Al-Assaf, "Prediction of the Fatigue Life of Unidirectional Glass Fiber/Epoxy Composite Laminate Using Different Neural Network Paradigms", Composite Structures, Vol.55, pp. 239-246, 2002

[10] H. El Kadi, Y. Al-Assaf, "Engery-Based Fatigue Life Prediction Fiberglass/Epoxy Composites Using Modular Neural Networks”, Composite Structures,Vol.57, pp. 85-89, 2002.

[11] S.W. Choi, E.J. Song, and H.T. Hahn, "Prediction of Fatigue Damage Growth in Notched Composite Laminates Using an Artificial Neural Network”, Composites Science and Technology, Vol.63, pp. 661-675, 2003.

[12] V.N. Vapnik "The Nature of Statistical Learning Theory” Springer-Verlag, New York, 1995

[13] V.N. Vapnik, S.E. Golowich, and A.J. Smola "Support Vector Method for Function Approximation, Regression Estimation, and Signal Processing”, Advance in Neural Information Processing Systems, Vol. 9, pp. 281287, 1996.

[14] F.E.H. Tay, L. Cao, “Application of Support Vector Machines in Financial Time Series Forecasting”, Omega, Vol.29, pp. 309-317, 2001.

[15] W.Wang, Zz. Xu, and J.W. Lu, "Three Improved Neural Network Models for Air Quality Forecasting”, Engineering Computations, Vol.20, pp. 192-210, 2003.

[16] M.A. Mohandes, T.O. Halawani, S. Rehman, and A.A. Hussain, A.A., "Support Vector Machines for Wind Speed Prediction”, Renewable Energy,Vol.29, pp. 939-947, 2004.

[17] P.F. Pai, "System Reliability Forecasting by Support Vector Machines with Genetic Algorithms", Mathematical And Computer Modeling, Vol. 43, pp.262-274. 2006.

[18] P.F. Pai, W.C. Hong, "Support Vector Machines With Simulated Annealing Algorithms in Electricity Load Forecasting”,Energy Conversion And Management Vol.46, pp. 2669-2688, 2005.

[19] P.F. Pai, W.C. Hong, "An Improved Neural Network Model in Forecasting Tourist Arrivals", Annals of Tourism Research, Vol.32, No.4, pp.1138-1141, 2005.

[20] S. Amari, S. Wu, "Improving Support Vector Machine Classifiers by Modifying Kernel Functions”, Neural Networks, Vol. 12. pp. 783789, 1999.

[21] G. Box, G. .M. Jenkins, "Time Series Analysis: Forecasting and Control", Holden-Day, San Francisco,1976.

[22] D.A. Specht, “A General Regression Neural Network", IEEE Transactions on Neural Networks", Vol. 2, pp. 568-576, 1991 